University of Nebraska - Lincoln

DigitalCommons@University of Nebraska - Lincoln

April 1973

\title{
Quasi-Landau spectrum of a hydrogen like atom in a high magnetic field
}

Anthony F. Starace

University of Nebraska-Lincoln, astarace1@unl.edu

Follow this and additional works at: https://digitalcommons.unl.edu/physicsstarace

Part of the Physics Commons

Starace, Anthony F., "Quasi-Landau spectrum of a hydrogen like atom in a high magnetic field" (1973). Anthony F. Starace Publications. 122.

https://digitalcommons.unl.edu/physicsstarace/122

This Article is brought to you for free and open access by the Research Papers in Physics and Astronomy at DigitalCommons@University of Nebraska - Lincoln. It has been accepted for inclusion in Anthony F. Starace Publications by an authorized administrator of DigitalCommons@University of Nebraska - Lincoln. 


\title{
Quasi-Landau spectrum of a hydrogen like atom in a high magnetic field
}

\author{
Anthony F. Starace \\ Department of Physics, Imperial College of Science and Technology, London SW7 \\ Current address: Behlen Laboratory of Physics, The University of Nebraska-Lincoln, \\ Lincoln, Nebraska 68508.
}

\begin{abstract}
A two dimensional semiclassical approximation is used to examine the $\sigma$ polarization spectrum of a hydrogen like atom in a strong magnetic field. Resonances above the zero field ionization limit are found and their energy separations are computed over the region from the ionization limit to $40 \hbar \omega_{\mathrm{c}}$ above, where $\omega_{\mathrm{c}}$ is the cyclotron frequency, for magnetic fields of $10,17,25,32,40$ and $47 \mathrm{kG}$. At the ionization limit the separation between resonances is found to be $1.500 \hbar \omega_{\mathrm{c}}$, independent of magnetic field strength, which is in agreement with a separation of $1.5 \hbar \omega_{\mathrm{c}}$ observed experimentally by Garton and Tomkins (1969) in the sigma polarization spectrum of barium in a magnetic field of $24 \mathrm{kG}$. The energy separations are found to decrease above the ionization limit toward the value $\hbar \omega_{c}$, which is characteristic of the levels of a free electron in a homogeneous magnetic field.
\end{abstract}

\section{Introduction}

A recent experiment on the 6s shell photoabsorption spectrum of barium in a magnetic field of $24 \mathrm{kG}$ (Garton and Tomkins 1969) shows diffuse resonances above the zero field threshold in $\sigma$ polarization. In the vicinity of the threshold these resonances have a separation of $1.5 \hbar \omega_{\mathrm{c}}$, where $\omega_{\mathrm{c}}$, is the cyclotron frequency. No resonances above the threshold were found in polarization. In addition, the experimental data below the threshold show in far greater detail the striking effects of configuration mixing that were first observed by Jenkins and Segre (1939) in the alkalis. These latter experiments were interpreted theoretically by Schiff and Snyder (1939). Recently Edmonds (1970) has indicated possible approximate methods for calculating theoretically the newly observed experimental spectrum for barium, both above and below the threshold. In particular, Edmonds (1970) suggests that the novel $\sigma$ polarization resonances above the threshold, which he has named "quasi-Landau" resonances, may be understood within the framework of a two dimensional, semiclassical approximation. This approximation has been developed by solid state physicists for application to the exciton spectrum of semiconductors in high magnetic fields (see the reviews by Hasegawa, 1969 and by Baldereschi and Bassani, 1970). Its applicability and predictions for atomic photoabsorption experiments such as that of Garton and Tomkins (1969) are the subject of this paper.

We present below two dimensional, semiclassical calculations for the $\sigma$ polarization spectrum of a hydrogen like atom in a strong magnetic field. We obtain from these calculations the energy separation of neighbouring quasi-Landau levels over the energy region from the ionization limit to $40 \hbar \omega_{\mathrm{c}}$ above using magnetic fields of $10,17,24$, 32,40 and $47 \mathrm{kG}$. Our results are meant for application to any atomic system since the 
details of the ionic core, which we have ignored, are not expected to affect the energy separations over the energy range considered. The present theoretical calculations represent an attempt to predict the results of forthcoming experiments by Garton and Tomkins (Garton 1972, private communication) on the spectrum of the quasi-Landau resonances over the energy range considered here and for the magnetic fields used in these calculations.

\section{Characteristics of the effective potential}

The spin independent hamiltonian for the valence electron of a hydrogen like atom in a uniform magnetic field directed along the $z$ axis has cylindrical symmetry. The invariant quantum numbers describing the state of the electron are the magnetic quantum number $M$ and the parity. Therefore, we write the wavefunction of the electron in cylindrical co-ordinates as :

$$
\psi(\rho, \phi, z)=\rho^{-1 / 2} f(\rho, z) \exp (\mathrm{i} M \phi) .
$$

In equation (1) we have factored out $\rho^{-1 / 2}$ in order to eliminate first derivatives from equation (2) below, which is obtained from the Schrödinger equation for $\psi(\rho, \phi, z)$ (Landau and Lifshitz 1965):

$$
\frac{\partial^{2} f(\rho, z)}{\partial \rho^{2}}+\frac{\partial^{2} f(\rho, z)}{\partial z^{2}}+\frac{2 m}{\hbar^{2}}\left\{E^{\prime}-V(\rho, z)\right\} f(\rho, z)=0
$$

where

$$
\begin{aligned}
& V(\rho, z) \equiv \frac{\hbar^{2}}{2 m} \frac{\left(M^{2}-\frac{1}{4}\right)}{\rho^{2}}-\frac{\mathrm{e}^{2}}{\left(\rho^{2}+z^{2}\right)^{1 / 2}}+\frac{1}{2} \mathrm{~m} \alpha^{2} \rho^{2} \\
& E^{\prime}=E-x \hbar M \quad \alpha=\frac{e H}{2 m c} .
\end{aligned}
$$

Here $H$ is the magnetic field strength, $E$ is the electron energy, and $E^{\prime}$ is the energy $E$ shifted by $-\alpha \hbar M$, which is the energy of the electron's orbital magnetic moment in the magnetic field. The three terms comprising the effective potential energy $V(\rho, z)$ are respectively the centrifugal potential (where the $-\frac{1}{4}$ in the numerator arises only because $\rho^{-1 / 2}$ was factored out in equation(1)), the Coulomb potential and the quadratic Zeeman energy. We have explicitly indicated all physical constants in the above equation to facilitate discussion later. Below, however, we shall for simplicity use atomic units (ie $e=m=\hbar=1$ ) unless noted otherwise.

The eigenfunctions $f_{n}$ and eigenvalues $E_{n}^{\prime}$ of equation (2) are difficult to obtain because the effective potential $V(\rho, z)$ is nonseparable. This potential, which has been studied in detail by Gajewski (1970), is the superposition of the spherically symmetric Coulomb potential and the cylindrically symmetric centrifugal and quadratic Zeeman energies. Briefly, its main features are:

(i) For all energies, motion in the $\rho$ direction is bounded for large $\rho$ by the quadratic Zeeman term and for small $\rho$ (when $M \neq 0$ ) by the centrifugal term.

(ii) For energies $E^{\prime} \gtrsim 0$ (where $E^{\prime}=0$ is the first ionization limit of the atom in the absence of a magnetic field) it is possible for the electron to escape to infinity by moving in a trajectory parallel to the $z$ axis such that $E^{\prime}-V(\rho, z) \geqslant 0$. This motion in $z$ is governed mainly by the Coulomb field. 
(iii) Thus for $E^{\prime} \gtrsim 0$ and $M \neq 0$ the equipotential $V(\rho, z)=E^{\prime}$ has the form of a pipe parallel to the $z$ axis with a bulge at $z=0$ due to the attractive, spherically symmetric Coulomb potential. Electrons with energy $E^{\prime} \gtrsim 0$ may escape by moving along the $z$ axis through one of the ends of the equipotential 'pipe'.

As an aside, note that the ionization limit in the presence of a magnetic field differs from the field-free ionization limit when $M \neq 0$ by an amount equal to the minimum value of $V(\rho, z=\infty)$. For fields of the order of $10^{12} \mathrm{G}$, for example, the ionization limit is raised considerably above the zero field limit (see Cohen et al 1970). For the kilogauss magnetic fields considered in this paper, however, this difference is negligible and is ignored.

These features of the effective potential $V(\rho, z)$ allow a conceptually simple interpretation of the results of Garton and Tomkins (1969) for the energy region above the ionization limit. Consider the operator for an electric dipole transition in the case of a one electron atom,

$$
Q_{q}^{1}=r C_{q}^{1}(\Omega)
$$

where $(r, \Omega)$ are the spherical coordinates of the electron and where $C_{q}^{1}(\Omega)$ is a tensor proportional to the spherical harmonic $Y_{1 q}(\theta, \phi)$ (Shore and Menzel 1968). In a $\pi$ polarization transition $q=0$ and hence the electron magnetic quantum number $M$ does not change during the transition, that is $\Delta M=0$. Since $C_{0}^{1}$ depends on $\theta$ as $\cos \theta$, in a $\pi$ polarization transition the final state of the electron is most probably located along the $z$ axis near either $\theta=0^{\circ}$ or $\theta=180^{\circ}$. (This corresponds to the classical result that in a $\pi$ polarization transition the radiating electron oscillates along the $z$ axis.) In a $\sigma$ polarization transition, however, $q= \pm 1$ and the magnetic quantum number $M$ changes, that is $\Delta M= \pm 1$. Since $C_{ \pm 1}^{1}$ depends on $\theta$ as $\sin \theta$, in a $\sigma$ polarization transition the final state of the electron is most probably located in the plane perpendicular to the $z$ axis defined by $\theta=90^{\circ}$ or $z=0$. (This corresponds to the classical result that in a $\sigma$ polarization transition the radiating electron oscillates in the $z=0$ plane.) Hence the electron's motion in the final state is bound even for $E^{\prime}>0$ in accordance with experiment. Note that these remarks are independent of the initial or final value of $M$; it is the dipole transition operator which determines the orientation of the final state of the electron and not the final state value of $M$.

Since the spherically symmetric Coulomb potential couples motion in $\rho$ with that in $z$, eventually an electron which is trapped in one of the $\sigma$ polarization resonances above the threshold 'autoionizes' into an orbit along the $z$ axis (with the same value of $M$ ) and escapes. Since the Coulomb potential is strongest near the origin, the autoionization transition matrix elements will be sensitive to the electron wavefunction near the origin. Hence the shape of the autoionizing $\sigma$ polarization resonances depends on the details of the electron-ion interaction, which will vary from atom to atom. The energy spacings between the $\sigma$ polarization resonances, as we shall see below, are determined by the effective potential $V(\rho, z=0)$ over a very large range of the coordinate $\rho$. Thus the energy spacings of the $\sigma$ polarization resonances are independent of the details of the electron-ion interaction and will have the same values for all atoms.

\section{3. $\sigma$ polarization spectrum in the two dimensional approximation}

For the reasons above we have computed the energy spacings of the quasi-Landau levels in the following two dimensional approximation. We consider the electron's motion in 
the $(\rho, \phi)$ plane to be governed by the effective potential $V(\rho, z=0)$. We replace $f(\rho, z)$ by $f(\rho)$, which satisfies the equation

$$
\frac{\mathrm{d}^{2} f(\rho)}{\mathrm{d} \rho^{2}}+\left(2 E^{\prime}-\frac{\left(M^{2}-\frac{1}{4}\right)}{\rho^{2}}+\frac{2}{\rho}-\alpha^{2} \rho^{2}\right) f(\rho)=0 .
$$

Since we are concerned with highly excited levels (ie large $n$ ) we determine the eigenvalues of equation 3 by means of the Bohr-Sommerfeld quantization condition

$$
\int_{\rho_{1}}^{\rho_{2}}\left(2 E^{\prime}-\frac{M^{2}}{\rho^{2}}+\frac{2}{\rho}-\alpha^{2} \rho^{2}\right)^{1 / 2} \mathrm{~d} \rho=\left(n+\frac{1}{2}\right) \pi
$$

where $\rho_{1}$ and $\rho_{2}$ are the two real roots of the integrand. Note that the centrifugal potential in equation (4) has been altered from that in equation (3) in order to apply the one dimensional WKB approximation to our two dimensional problem (Akimoto and Hasegawa 1967).

The quantum number $n$ is equal to the number of nodes of the eigenfunction $f_{n}(\rho)$ belonging to the energy eigenvalue $E_{n}^{\prime}$. In the limiting cases of (i) pure Coulomb field and (ii) pure homogeneous magnetic field, $n$ is simply related to (i) the principal quantum number and (ii) the Landau quantum number (Hasegawa 1969). In the case of combined Coulomb and magnetic fields, evaluation of equation (4) for $E^{\prime} \simeq 0$ gives $n=69$ for a magnetic field of $10 \mathrm{kG}$ and $n=41$ for a magnetic field of $47 \mathrm{kG}$. These numbers indicate that, in the two dimensional approximation, there are no longer an infinite number of levels below the ionization limit and that the stronger the magnetic field the greater the number of levels raised above the limit. Whether or not the absolute values of $n$ have any correspondence with experiment is unclear, especially since for values of $E^{\prime}$ below the ionization limit the electron's motion is generally neither two dimensional nor semiclassical.

The spacing between calculated quasi-Landau levels in the region $E^{\prime} \geqslant 0$ should, however, correspond with experiment. This spacing is equal to $\mathrm{d} E / \mathrm{d} n$, which is obtained by differentiating equation 4 ,

$$
\left(\frac{\mathrm{d} E}{\mathrm{~d} n}\right)^{-1}=\frac{1}{\pi} \int_{\rho_{1}}^{\rho_{2}}\left(2 E^{\prime}-\frac{M^{2}}{\rho^{2}}+\frac{2}{\rho}-\alpha^{2} \rho^{2}\right)^{-1 / 2} \mathrm{~d} \rho .
$$

Again, $\rho_{1}$ and $\rho_{2}$ are the two real roots of the expression in brackets. For all fields and energies considered here $\rho_{1} \simeq 0.5 \mathrm{Bohr}$ for $M=1$. At the ionization limit $\left(E^{\prime}=0\right)$, $\rho_{2}=2.7 \times 10^{3}$ Bohr for $H=47 \mathrm{kG}$ and $\rho_{2}=7.6 \times 10^{3}$ Bohr for $H=10 \mathrm{kG}$, also for $M=1$. Over these large ranges of the coordinate $\rho$ the electron's motion is certainly quasiclassical. In the limiting case of no Coulomb field equation (5) gives the result (written in ordinary units)

$$
\left(\hbar \omega_{\mathrm{c}}\right)^{-1}(\mathrm{~d} E / \mathrm{d} n)=1
$$

This indicates that the energy levels are equally spaced by an amount equal to the cyclotron energy, $\hbar \omega_{c}$. This is the usual property of the ordinary Landau levels for a free electron in motion perpendicular to a homogeneous magnetic field.

In figure 1 we have plotted the energy spacing, $\left(\hbar \omega_{c}\right)^{-1}(\mathrm{~d} E / \mathrm{d} n)$, against the energy relative to the ionization limit, $E^{\prime} / \hbar \omega_{\mathrm{c}}$, for six values of the magnetic field ranging from 10 to $47 \mathrm{kG}$. In these calculations we have used $M=1$ in order to correspond with the experiment of Garton and Tomkins (1969) and we do not expect the results to be very sensitive to the particular value of $M$. For $E^{\prime}=0$, the energy spacing is 1.500 for all six 


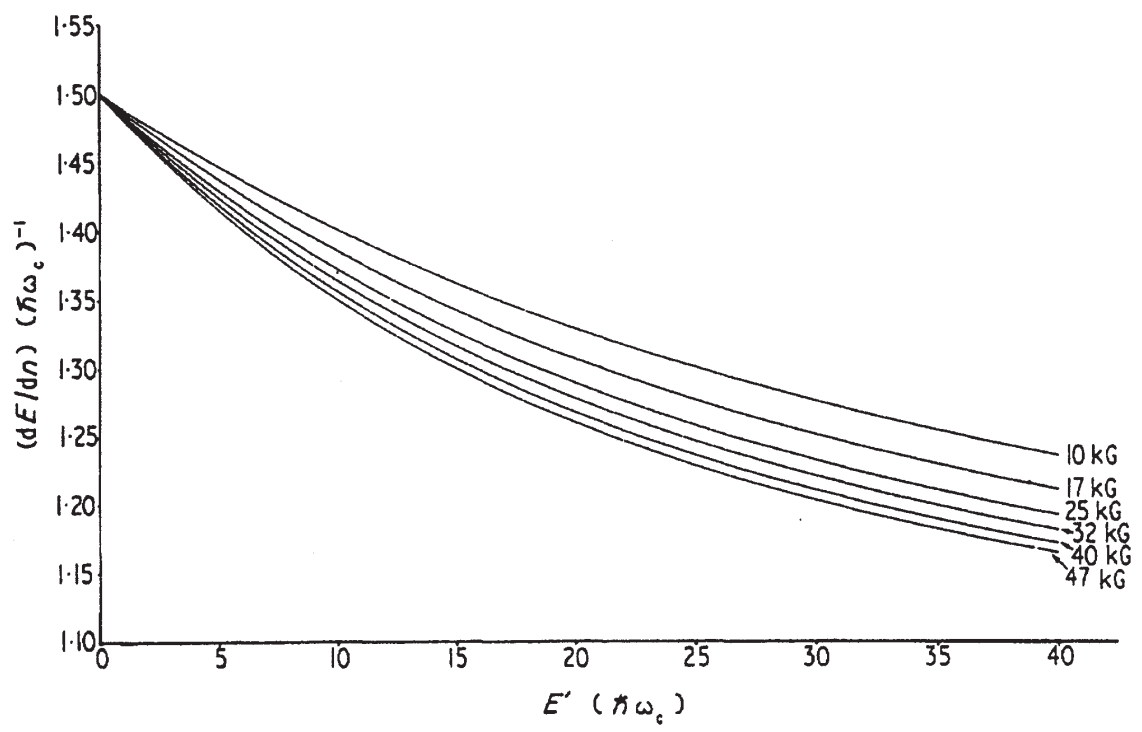

Figure 1. Plot of the energy separation $d E / d n$ against energy $E^{\prime}$ above the threshold for six values of the magnetic field, $H=10 \mathrm{kG}, 17 \mathrm{kG}, 25 \mathrm{kG}, 32 \mathrm{kG}, 40 \mathrm{kG}, 47 \mathrm{kG}$. Both $\mathrm{d} E / \mathrm{d} n$ and $E^{\prime}$ are in units of the cyclotron energy, $\hbar \omega_{c}=h(e H / m c)$. As $E^{\prime} \rightarrow \infty_{1}\left(\hbar \omega_{c}\right)^{-1}(\mathrm{~d} E / \mathrm{d} n) \rightarrow 1$.

values of $H$. This result is in complete agreement with the energy spacing of 1.5 for $H=24 \mathrm{kG}$ found by Garton and Tomkins (1969) in barium. It also agrees with the analytic result of $3 / 2$ obtained from equation (5) for $E^{\prime}=0$ when the centrifugal term, which has a factor $\hbar^{2}$ (see equation (2)), is ignored (Edmonds 1972, private communication).

For $0<E^{\prime}<\infty$, figure 1 shows that the energy spacing decreases as $E^{\prime}$ increases and that the spacing decreases faster for stronger magnetic fields. In the limit that $E^{\prime} \rightarrow \infty$ the spacing tends to 1 , which is the pure magnetic field result. These results on the energy and field dependence of $\mathrm{d} E / \mathrm{d} n$ will be tested by forthcoming experiments of Garton and Tomkins (Garton 1972, private communication).

\section{Conclusions}

We have used a two dimensional, semiclassical approximation to examine the energy and magnetic field dependence of the energy separations between neighbouring $\sigma$ polarization quasi-Landau resonances. In contrast to a theoretical hypothesis of Schiff and Snyder (1939), these separations have been found to be strongly affected by the Coulomb field of the ion since the computed values differ markedly from those characteristic of a free electron in a homogeneous magnetic field. Our computed energy separation for the quasiLandau levels at the ionization limit is $1.500 \hbar \omega_{c}$ independent of magnetic field strength. This result is in excellent agreement with the experiment of Garton and Tomkins (1969).

\section{Acknowledgments}

I am grateful to Dr A R Edmonds, Professor W R S Garton, and Dr R C M Learner for 
their critical readings of the manuscript. I also wish to thank Dr R W Lee for the use of his computer program to plot the graph in figure 1.

\section{References}

Akimoto O and Hasegawa H 1967 J. Phys. Soc. Japan 22 181-91

Baldereschi A and Bassani F 1970 Proceedings of the 10th Int. Conf. on the Physics of Semiconductors, Cambridge, Massachusetts, August 17-2l ed S P Keller, J C Hensel and F Stern (Washington DC: US Atomic Energy Commission) pp 191-6

Cohen R, Lodenquai J and Ruderman M 1970 Phys. Rev. Lett. 25 467-9

Edmonds A R 1970 J. Phys. Paris 31 Colloque C4 71-4

Gajewski R 1970 Physica 47 575-95

Garton W R S and Tomkins F S 1969 Astrophys. J. 158 839-45

Hasegawa H 1969 Physics of Solids in Intense Magnetic Fields ed E D Haidemenakis (New York: Plenum) pp $247-70$

Jenkins F A and Segrè E 1939 Phys. Rev. 55 52-8

Landau L D and Lifshitz E M 1965 Quantum Mechanics 2nd Ed. (London: Pergamon) p 426

Schiff L I and Snyder H 1939 Phys. Rev 55 59-63

Shore B W and Menzel D H 1968 Principles of Atomic Spectra (New York: Wiley) pp 164 and 442 